NBER WORKING PAPER SERIES

INDETERMINACY IN A FORWARD LOOKING REGIME SWITCHING MODEL

\author{
Roger E. A. Farmer \\ Daniel F. Waggoner \\ Tao Zha \\ Working Paper 12540 \\ http://www.nber.org/papers/w12540 \\ NATIONAL BUREAU OF ECONOMIC RESEARCH \\ 1050 Massachusetts Avenue \\ Cambridge, MA 02138 \\ September 2006
}

We thank Troy Davig, Jordi Gali, Eric Leeper, Julio Rotemberg, Tom Sargent, Chris Sims, Lars Svensson, Eric Swanson, Noah Williams and Michael Woodford for helpful discussions. The views expressed herein do not necessarily reflect those of the Federal Reserve Bank of Atlanta or the Federal Reserve System. Farmer acknowledges the support of NSF grant \#SES 0418074. The views expressed herein are those of the author(s) and do not necessarily reflect the views of the National Bureau of Economic Research.

(C) 2006 by Roger E. A. Farmer, Daniel F. Waggoner, and Tao Zha. All rights reserved. Short sections of text, not to exceed two paragraphs, may be quoted without explicit permission provided that full credit, including $\odot$ notice, is given to the source. 
Indeterminacy in a Forward Looking Regime Switching Model

Roger E. A. Farmer, Daniel F. Waggoner, and Tao Zha

NBER Working Paper No. 12540

September 2006

JEL No. E31,E4,E52

\begin{abstract}
$\underline{\text { ABSTRACT }}$
This paper is about the properties of Markov switching rational expectations (MSRE) models. We present a simple monetary policy model that switches between two regimes with known transition probabilities. The first regime, treated in isolation, has a unique determinate rational expectations equilibrium and the second contains a set of indeterminate sunspot equilibria. We show that the Markov switching model, which randomizes between these two regimes, may contain a continuum of indeterminate equilibria. We provide examples of stationary sunspot equilibria and bounded sunspot equilibria which exist even when the MSRE model satisfies a 'generalized Taylor principle'. Our result suggests that it may be more difficult to rule out non-fundamental equilibria in MRSE models than in the single regime case where the Taylor principle is known to guarantee local uniqueness.

Roger E. A. Farmer

UC, Los Angeles

Department of Economics

Box 951477

Los Angeles, CA 90095-1477

and NBER

rfarmer@econ.ucla.edu

Daniel F. Waggoner

Federal Reserve Bank of Atlanta

1000 Peachtree Street N.E.

Atlanta Georgia 30309-4470

dwaggoner@frbatlanta.org

Tao Zha

Federal Reserve Bank of Atlanta 1000 Peachtree Street N.E.

Atlanta, Georgia 30309-4470

tzha@earthlink.net
\end{abstract}




\title{
INDETERMINACY \\ IN A FORWARD LOOKING REGIME SWITCHING MODEL
}

\author{
ROGER E. A. FARMER, DANIEL F. WAGGONER, AND TAO ZHA
}

\begin{abstract}
This paper is about the properties of Markov switching rational expectations (MSRE) models. We present a simple monetary policy model that switches between two regimes with known transition probabilities. The first regime, treated in isolation, has a unique determinate rational expectations equilibrium and the second contains a set of indeterminate sunspot equilibria. We show that the Markov switching model, which randomizes between these two regimes, may contain a continuum of indeterminate equilibria. We provide examples of stationary sunspot equilibria and bounded sunspot equilibria which exist even when the MSRE model satisfies a 'generalized Taylor principle'. Our result suggests that it may be more difficult to rule out non-fundamental equilibria in MRSE models than in the single regime case where the Taylor principle is known to guarantee local uniqueness.
\end{abstract}

\section{INTRODUCTION}

Work by Richard Clarida, Jordi Galí and Mark Gertler (2000) has stimulated recent interest in models where monetary policy may occasionally change between a passive regime in which there exists an indeterminate set of sunspot equilibria and an active regime in which equilibrium is unique. Models of this kind are inherently non-linear since the parameters of the model are represented by elements of a Markov chain.

Papers in the literature on nonlinear rational expectations models typically compute a solution to functional equations using numerical methods, but not much is known about the analytical properties of these equations. In an important paper, Lars Svensson and Noah Williams (SW) (2005) have proposed an algorithm for solving Markov switching rational expectations (MSRE) models. Our computational experiments suggest that the SW algorithm will find the unique value of the minimum-statevariable (MSV) when it exists but it may also converge to one of a set of indeterminate equilibria (Farmer, Waggoner, Zha (2006, Appendix 2)). Obtaining a complete set of

Date: September 27, 2006.

Key words and phrases. Policy rule, inflation, serial dependence, multiple equilibria, regime switching.

We thank Troy Davig, Jordi Galí, Eric Leeper, Julio Rotemberg, Tom Sargent, Chris Sims, Lars Svensson, Eric Swanson, Noah Williams and Michael Woodford for helpful discussions. The views expressed herein do not necessarily reflect those of the Federal Reserve Bank of Atlanta or the Federal Reserve System. Farmer acknowledges the support of NSF grant \#SES 0418074. 
indeterminate equilibria even for a simple MSRE model is a much more difficult problem, and to the best of our knowledge there are no systematic methods to accomplish this task.

The distinction between the linear RE model and the MSRE model is subtle but important and the conditions for existence and boundedness of a unique solution are different in the two cases. In this paper we study a simple Markov-switching model of inflation that combines two purely forward-looking rational expectations models. The first one has a unique determinate equilibrium and the second is associated with a set of indeterminate sunspot equilibria. The MSRE model switches between the two models with transition probabilities governed by a Markov chain.

Within the MSRE environment we first establish the existence of an MSV equilibrium for almost all values of the transition probabilities. We go on to discuss alternative definitions of stationarity for non-linear models and we argue that meansquare stability is an appropriate and appealing concept. We then show through a series of examples that there exists a set of mean-square-stable sunspot equilibria for large open sets of the model's parameter values. Our results imply that the existence of stationary sunspot equilibria in MRSE models is a pervasive phenomenon that cannot be ruled out in all regimes by the actions of the policy maker in a single regime.

\section{The MODEL}

Following Robert King (2000) and Michael Woodford (2003), we study a simple flexible price model in which the central bank can affect inflation but not the real interest rate. In this model, the Fisher equation links the real interest rate, $r_{t}$, and the nominal interest rate, $R_{t}$, by the equation,

$$
R_{t}=E_{t}\left[\pi_{t+1}\right]+r_{t}
$$

where $E_{t}$ is the mathematical expectation at date $t$ and $\pi_{t+1}$ is the inflation rate at date $t+1$. The central bank sets the time-varying rule

$$
R_{t}=\phi_{\xi_{t}} \pi_{t}-\kappa_{\xi_{t}} \varepsilon_{t}
$$

where $\xi_{t}$ is a two-state Markov process with transition probabilities $\left(p_{i, j}\right)$ and $p_{i, j}$ is the probability of transiting from state $j$ to state $i$. The stochastic process $\left\{\varepsilon_{t}\right\}_{t=1}^{\infty}$ is independently distributed with mean zero and unit variance and is independent of $\left\{\xi_{t}\right\}_{t=1}^{\infty}$. Substituting Eq. (2) into Eq. (1) gives the following forward-looking inflation process

$$
\phi_{\xi_{t}} \pi_{t}=E_{t}\left[\pi_{t+1}\right]+r_{t}+\kappa_{\xi_{t}} \varepsilon_{t} .
$$

We assume that the real interest rate evolves exogenously according to

$$
r_{t}=\rho r_{t-1}+\nu_{t}
$$

where $|\rho|<1$ and $\left\{\nu_{t}\right\}_{t=1}^{\infty}$ is independently distributed with zero mean and finite variance and is independent of $\left\{\xi_{t}\right\}_{t=1}^{\infty}$ and $\left\{\varepsilon_{t}\right\}_{t=1}^{\infty}$. 


\section{An Appropriate Equilibrium Concept}

Much of the previous work on dynamic stochastic general equilibrium theory has been concerned with constant parameter models. A typical way to proceed is to specify preferences, technology and endowments and to make an explicit assumption about the nature of stochastic shocks. Sometimes it is possible to specify an environment in which, in the absence of shocks, there exists a unique stationary perfect foresight equilibrium. An example is the single agent real business cycle model. When the stationary equilibrium is unique it may be possible to approximate a stochastic rational expectations equilibrium by linearizing the non-stochastic model around the steady state and solving for an approximate stochastic rational expectations equilibrium. For this solution to be asymptotically valid the stochastic shocks must be bounded. Boundedness is necessary to keep the system close to the non-stochastic steady state - the only point in the state space for which the linear approximation is exact.

The non-stochastic dynamics of a perfect foresight linear model are completely characterized by the roots of the characteristic polynomial of a first order matrix difference equation. When all of these roots lie within the unit circle, the stochastic process is stationary and, as a consequence, it is possible to prove theorems which assert that as the variance of the shocks goes to zero, the approximation error vanishes. One would like to prove a similar theorem for the Markov switching model but since the model is inherently non-linear, this is impossible. To make progress with models of this kind one needs an appropriate equilibrium concept different from that used for linear RE models. Specifically, one would like to describe solutions that are stationary in a Markov-switching model. In this paper we adopt a solution that is widely used by engineers and control theorists, that of mean square stability. ${ }^{1}$

\section{Determinate And Indeterminate Solutions and the Taylor PRINCIPLE}

In single regime models there is a simple test for uniqueness that involves counting unstable roots and nonpredetermined variables. ${ }^{2}$ Rational expectations equilibrium is unique if the number of non-predetermined variables is equal to the number of unstable roots. This root counting condition lies behind the Taylor principle; that monetary equilibrium will be locally unique if the central bank follows a monetary policy in which it raises the interest rate in response to inflation with a response coefficient greater than one in absolute value.

In an innovative paper, Troy Davig and Eric Leeper $(2005,2006)$ have tackled the question of how to think about indeterminacy in models of regime switching. Their

\footnotetext{
${ }^{1}$ The reader is referred to Costa Fragoso and Marques (2004). For linear models, one should note that mean square stability is the same as the conventional definition of stationarity.

${ }^{2}$ As Sims (2001) points out, this test does not always work and the exact condition is more complicated.
} 
idea is to find a condition, similar to the Taylor principle, that applies to MSRE models. Davig and Leeper define determinacy to mean the existence of a unique bounded solution to a stochastic linear system. By imposing the restriction that the interest rate coefficient of the Taylor rule must be positive, they find a condition they call a 'long-run Taylor principle' that involves a combination of interest rate response coefficients and transition probabilities. If this condition holds there exists a unique bounded equilibrium. If it fails there may be multiple equilibria driven by non-fundamental shocks.

We have two criticisms of the Davig-Leeper result. First, we think the positivity restriction is not merely for mathematical convenience, but rather an indication that there does not exist in general an equivalence between the existence of a unique bounded equilibrium for a MSRE model and the generalized Taylor principle derived from the linear RE counterpart. Economically, even if one believes that it is appropriate for a benevolent policy maker to choose a positive value for the interest rate response coefficient to inflation in the Taylor rule, one still cannot rule out the possibility that an incompetent or ill-informed policy maker might react differently. Moreover, Rotemberg and Woodford (1999a, Page 83) have shown that the optimal Taylor rule may involve a negative value of this parameter. In Section VII we exploit the fact that one or more regimes may be associated with a negative value for the inflation response coefficient to provide an example where there exist multiple bounded sunspot equilibria even when the generalized Taylor principle is satisfied.

Second, we think that the boundedness criterion is too strong. To see why, note that stationarity and boundedness are equivalent for a stochastic linear system when all the shocks are restricted to be bounded. In practice, the distributions of the shocks are often assumed to be unbounded (e.g., normal or gamma distributions). But as long as the stochastic process is stationary, the approximation around the steady state remains reasonable. Similarly, the mean-square-stable process for a Markov-switching model is stable around the steady state, which is what one needs for reasonable approximations to the underlying economic environment.

\section{The MSV Solution}

In a companion to this paper, Farmer Waggoner and Zha (2006) suggest MSV solutions as a way to make progress in the study of MSRE models. The purpose of this section is to show that a solution of this kind exists to the model defined above when inflation is chosen to be the state variable. To prove this, and to derive the MSV solution in terms of $\pi_{t}$, we need to rule out a knife edge parameter case. That is the purpose of the following assumption.

Assumption 1. The matrix

$$
\left[\begin{array}{cc}
\phi_{1}-\rho p_{1,1} & -\rho p_{2,1} \\
-\rho p_{1,2} & \phi_{2}-\rho p_{2,2}
\end{array}\right]
$$


is invertible.

Given assumption 1 we can prove the following proposition.

Proposition 1. Let

$$
\left[\begin{array}{l}
g_{1} \\
g_{2}
\end{array}\right]=\left[\begin{array}{cc}
\phi_{1}-\rho p_{1,1} & -\rho p_{2,1} \\
-\rho p_{1,2} & \phi_{2}-\rho p_{2,2}
\end{array}\right]^{-1}\left[\begin{array}{l}
1 \\
1
\end{array}\right] .
$$

Under Assumption 1,

$$
\pi_{t}=g_{\xi_{t}} r_{t}+\frac{\kappa_{\xi_{t}}}{\phi_{\xi_{t}}} \varepsilon_{t}
$$

is a solution to the model (3). ${ }^{3}$

Proof. The proof follows directly from Assumption 1 and the fact that

$$
E_{t}\left[g_{\xi_{t+1}}\right]=p_{1, \xi_{t}} g_{1}+p_{2, \xi_{t}} g_{2} \text {. }
$$

It follows from Theorems 3 and 4 in Farmer Waggoner and Zha (2006) that Eq. (6) is also the MSV solution. Following standard usage in the probability literature on Markov switching models (e.g., Costa Fragoso and Marques (2004)), we define stationarity to be the existence of limiting first and second moments and it follows directly from Proposition 1 and the stationarity of $r_{t}$ and $\varepsilon_{t}$ that the MSV solution is stationary.

Definition 1 (Mean Square Stability). A stochastic process $\left\{x_{t}\right\}_{t=1}^{\infty}$ is mean square stable if there exist real numbers $\mu$ and $\varphi$ such that

$$
\begin{aligned}
& \lim _{s \rightarrow \infty} E_{t}\left[x_{t+s}\right]=\mu, \\
& \lim _{s \rightarrow \infty} E_{t}\left[x_{t+s}^{2}\right]=\varphi .
\end{aligned}
$$

Mean square stability is stronger than the existence of a finite limit of first moments and it is the appropriate stability concept if one wants to conduct statistical inference. It is widely used in the engineering literature and has been used in an economic application, among others, by Svensson and Williams (2005). An alternative to mean square stability is covariance stationarity in the sense of Hamilton (1994), or asymptotic covariance stationarity - a slightly weaker condition. Asymptotic covariance stationarity implies mean square stability, although the converse is not true in general. However, for the class of solutions studied in this paper, Theorem 3.33 of Costa Fragoso and Marques (2004) implies that these two notions are equivalent. Because of this fact, we will refer to solutions that satisfy the mean-square stability criterion as "stationary".

Proposition 2. The MSV solution (6) is stationary.

\footnotetext{
${ }^{3}$ Our model is similar to that studied by Davig and Leeper (2005). The difference is that they use $E_{t}\left[r_{t+1}\right]$ in the Fisher equation whereas, following King (2000) and Woodford (2003), we use $r_{t}$.
} 
Proof. The proof follows directly from Definition 1.

Since the MSV solution exists for all the values of transition probabilities that satisfy Assumption 1, it does not depend on the ergodic nature of transition probabilities.

\section{A Class of Indeterminate Solutions}

Since the existence of a unique determinate solution is often viewed as a desirable feature of a model (Rotemberg and Woodford (1999b), King (2000)), we address the question: Is the MSV solution (6) unique in the class of all stationary solutions? Often the answer to this question is negative and we illustrate this point by first constructing a class of indeterminate solutions with the condition $\lim _{s \rightarrow \infty} E_{t}\left[x_{t+s}\right]<\infty$ as in the linear RE case. In the next section, we restrict solutions to stationary ones only. Our example is instructive since it suggests that the idea that MSRE models are either determinate or indeterminate may not be fruitful.

Our model contains two state variables, $\pi_{t}$ and $r_{t}$. However, all of the equilibria that we are interested in can be summarized a by a linear combination of these variables, defined as follows; ${ }^{4}$

$$
x_{t}=\pi_{t}-g_{\xi_{t}} r_{t} .
$$

Proposition 1 and Eq. (7) allow us to make the following change of variables.

Proposition 3. The inflation equation, Eq. (3), is equivalent to the following transformed equation in the variable $x_{t}$,

$$
x_{t}=\frac{1}{\phi_{\varepsilon_{t}}} E_{t}\left[x_{t+1}\right]+\frac{\kappa_{\xi_{t}}}{\phi_{\varepsilon_{t}}} \varepsilon_{t} .
$$

Proof. See Appendix 3.

Rearranging Eq. (8), one can write the following expressions for $x_{t+1}$ and $E_{t}\left[x_{t+1}\right]$,

$$
\begin{gathered}
x_{t+1}=\phi_{\varepsilon_{t}}\left(x_{t}-\frac{\kappa_{\xi_{t}}}{\phi_{\varepsilon_{t}}} \varepsilon_{t}\right)+\eta_{t+1}, \\
E_{t}\left[x_{t+1}\right]=\phi_{\varepsilon_{t}}\left(x_{t}-\frac{\kappa_{\xi_{t}}}{\phi_{\varepsilon_{t}}} \varepsilon_{t}\right),
\end{gathered}
$$

where $\eta_{t+1}$ is an expectational error such that $E_{t}\left[\eta_{t+1}\right]=0$.

Next, we make an assumption that characterizes the kind of central banker that acts in each regime. The idea is that the policy maker in regime 1 is an inflation hawk and the policy maker in regime 2 , an inflation dove. In line with the existing literature on the Taylor principle, we do not assume that the inflation response coefficients, $\phi_{1}$ and $\phi_{2}$, must be positive.

\footnotetext{
${ }^{4}$ Since $r_{r}$ is exogenous and stationary, and since we assume that $\xi_{t}$ is ergodic and that $g_{\xi_{t}} x_{t}$ is uncorrelated with $r_{t}, x_{t}$ will be stationary if and only if $\pi_{t}$ is stationary. Although we will work with $x_{t}$ directly, the reader should bear in mind that the behavior of the inflation variable $\pi_{t}$ can be recovered from Eq. (7).
} 
Assumption 2.

$$
\left|\phi_{1}\right|>1>\left|\phi_{2}\right|>0 \text {. }
$$

Assumption 2 implies that the two separate single-state models, where $\phi_{\xi_{t}}=\phi_{i}$ for all $t$, have different determinacy properties. If we impose the assumption that a solution must satisfy the additional boundary condition,

$$
\lim _{s \rightarrow \infty} E_{t}\left[x_{t+s}\right]=\mu<\infty,
$$

then the linear model with $\phi_{\xi_{t}}=\phi_{1}$ has a unique solution represented by the equation

$$
x_{t}=\frac{\kappa_{1}}{\phi_{1}} \varepsilon_{t},
$$

and the linear model with $\phi_{\xi_{t}}=\phi_{2}$ is associated with a continuum of indeterminate sunspot solutions of the form,

$$
x_{t}=\phi_{2} x_{t-1}-\kappa_{2} \varepsilon_{t-1}+\gamma_{t}
$$

where $\left\{\gamma_{t}\right\}_{t=1}^{\infty}$ is an independent stochastic process with zero mean and finite variance that is independent of $\left\{\nu_{t}\right\}_{t=1}^{\infty}$ and $\left\{\xi_{t}\right\}_{t=1}^{\infty}{ }^{5}$

Definition 2. A mean zero stochastic process $\left\{\eta_{t+1}\right\}_{t=1}^{\infty}$, and an initial condition $x_{1}$ generate a solution to Eq. (8) if the sequence $\left\{x_{t+1}\right\}_{t=1}^{\infty}$ defined by Eq. (9) satisfies the condition

$$
\lim _{s \rightarrow \infty} E_{t}\left[x_{t+s}\right]=\mu<\infty .
$$

Note that we define a solution to be a stochastic process with convergent first moments that satisfies Eq. (8) regime by regime and that transits between regimes according to the transition probabilities $\left(p_{i, j}\right)$. We now show how to construct a large class of processes $\left\{\left\{\eta_{t+1}\right\}_{t=1}^{\infty}, x_{1}\right\}$ that generate solutions to Eq. (9) and that are different from the MSV solution. We first rule out a pathological case in which our result breaks down.

Assumption 3. The transition matrix satisfies the condition,

$$
p_{2,2}>0
$$

This assumption rules out the case where the second regime is a reflecting state. Define

$$
x_{1}= \begin{cases}\frac{\kappa_{1}}{\phi_{1}} \varepsilon_{1} & \text { if } \xi_{1}=1 \\ \bar{x} \in \mathbb{R} & \text { if } \xi_{1}=2\end{cases}
$$

\footnotetext{
${ }^{5}$ Note that $\gamma_{t}$ could also be a function of $\varepsilon_{t}$ and may or may not contain a component that is independent of $\varepsilon_{t}$. Sunspot models of this kind are often interpreted as over-reaction to fundamentals.
} 
For $t \geq 1$, define $\eta_{t+1}$ by

$$
\eta_{t+1}=\left\{\begin{aligned}
\frac{\kappa_{1}}{\phi_{1}} \varepsilon_{t+1}, & \xi_{t}=1 \text { and } \xi_{t+1}=1, \\
\gamma_{t+1}+\frac{\kappa_{2}}{\phi_{2}} \varepsilon_{t+1}, & \xi_{t}=1 \text { and } \xi_{t+1}=2, \\
\frac{\kappa_{1}}{\phi_{1}} \varepsilon_{t+1}-\phi_{2}\left(x_{t}-\frac{\kappa_{2}}{\phi_{2}} \varepsilon_{t}\right), & \xi_{t}=2 \text { and } \xi_{t+1}=1, \\
\gamma_{t+1}+\frac{\kappa_{1}}{\phi_{1}} \varepsilon_{t+1}+\phi_{2} \frac{p_{1,2}}{p_{2,2}}\left(x_{t}-\frac{\kappa_{2}}{\phi_{2}} \varepsilon_{t}\right), & \xi_{t}=2 \text { and } \xi_{t+1}=2 .
\end{aligned}\right.
$$

Since $p_{2,2}>0$, the expectational errors $\left\{\eta_{t+1}\right\}_{t=1}^{\infty}$ are finite. Note that time begins at date 1 but the forecast errors begin with $\eta_{2}$. We now state the main result of our paper.

Proposition 4. The pair $\left\{\left\{\eta_{t+1}\right\}_{t=1}^{\infty}, x_{1}\right\}$, as defined in Eqs. (14) and (15), generates a solution to Eq. (8) for any arbitrary zero mean sequence $\left\{\gamma_{t+1}\right\}_{t=1}^{\infty}$. This indeterminate solution takes the following form:

$$
\begin{gathered}
x_{t+1}= \begin{cases}\frac{\kappa_{1}}{\phi_{1}} \varepsilon_{t+1}, & \text { if } \xi_{t}=1 \text { and } \xi_{t+1}=1, \\
\gamma_{t+1}+\frac{\kappa_{2}}{\phi_{2}} \varepsilon_{t+1}, & \text { if } \xi_{t}=1 \text { and } \xi_{t+1}=2 .\end{cases} \\
x_{t+1}= \begin{cases}\frac{\kappa_{1}}{\phi_{1}} \varepsilon_{t+1}, & \text { if } \xi_{t}=2 \text { and } \xi_{t+1}=1, \\
\gamma_{t+1}+\frac{\kappa_{2}}{\phi_{2}} \varepsilon_{t+1}+\frac{\phi_{2}}{p_{2,2}}\left(x_{t}-\frac{\kappa_{2}}{\phi_{2}} \varepsilon_{t}\right), & \text { if } \xi_{t}=2 \text { and } \xi_{t+1}=2 .\end{cases}
\end{gathered}
$$

Proof. See Appendix B.

A solution consists of an initial condition and a rule for updating expectations; this rule is implicitly defined by the endogenous shock process (15). Since the realization of $x_{1}$ is a function of the expectation, $E_{1}\left[x_{2}\right]$, the initial condition (14) imposed on the realization of $x_{1}$ is equivalent to the initial condition imposed on the expectation of $x_{2}$. If $\xi_{t}=1, E_{1}\left[x_{2}\right]=0$. If $\xi_{t}=2$, the initial condition $E_{1}\left[x_{2}\right]$ does not constrain $x_{1}$ and in this case, our definition of equilibrium picks an arbitrary initial condition, $\bar{x}$.

The former discussion is informative because it suggests why the search for a global uniqueness condition such as the generalized Taylor principle is unlikely to be successful. If the economy begins in state 1 , the regime of the inflation hawk, the value of inflation is pinned down by fundamentals. If instead the economy begins in state 2 , that is, if the inflation dove goes first, the initial value of inflation is unconstrained and there exist multiple self-fulfilling paths. Since this simple model contains no lagged state variables there is no history to constrain actions; all behavior is purely forward-looking and there is a sense in which the world begins again in every period. ${ }^{6}$ In regime 2 anything goes but, in regime 1, the economy must 'snap back' to a point pinned down by fundamentals.

\footnotetext{
${ }^{6}$ There may even be other equilibria of this model, that we have not explored, in which the existence of an inflation dove permits non-fundamental shocks to hit the system even in the regime of the inflation hawk.
} 


\section{Stationary Solutions}

In this section we illustrate our results by providing two examples of indeterminate solutions. The first example satisfies the mean-square stability condition so that the solution is stationary. The second example satisfies the more restricted Davig-Leeper definition of a stationary equilibrium for which the stationary invariant distribution is bounded. In both examples, we show that the generalized Taylor principle fails to hold.

We begin with a general characterization of stationary solutions based on Proposition 4 which implies that while in regime $2, x_{t}$ will be serially dependent and governed by the process:

$$
x_{t+1}=\frac{\phi_{2}}{p_{2,2}}\left(x_{t}-\frac{\kappa_{2}}{\phi_{2}} \varepsilon_{t}\right)+\frac{\kappa_{2}}{\phi_{2}} \varepsilon_{t+1}+\gamma_{t+1} .
$$

If $\left|\frac{\phi_{2}}{p_{22}}\right|$ is less than one, $x_{t}$ will be stationary in regime $2 .{ }^{7}$ But even if $\left|\frac{\phi_{2}}{p_{22}}\right|$ is greater than one, $x_{t}$ may still be globally stationary if the rate at which $x_{t}$ is increasing in the second regime is small relative to its expected duration. The following proposition gives the general condition for determining when $x_{t}$ is stationary.

Proposition 5. If the Markov chain is ergodic and $\left|\frac{\phi_{2}}{\sqrt{p_{2,2}}}\right|<1$, then the solutions to Model (8), given by Eqs. (14), (16), and (17), are mean-square-stable.

Proof. See Appendix C.

Notice that the stationarity of our constructed sunspot equilibrium depends only on the values of the parameters in the second regime and on the probability of staying in that regime. Notably, it does not depend on the probability of staying in the first (determinate) regime nor does it depend on the degree to which the inflation hawk (the policy maker in the determinate regime) follows an active policy.

Our first example, illustrated in Figure 1, displays the MSV solution in the first panel and the paths of inflation associated with two different sunspot equilibria in the second and third panels. These two sunspot equilibria are associated with two different parameterizations of the economy.

For both parameterizations we set $\rho=0.9, \phi_{1}=2.2, p_{1,1}=0.98$, and $p_{2,2}=0.995$. These parameter values correspond to monetary policy that would generate a unique equilibrium if the first regime were treated in isolation. The parameterizations differ by allowing alternative values of the interest rate coefficient in regime 2 . In one case we set $\phi_{2}=0.9951$ and in the other $\phi_{2}=0.9949$. In both cases monetary policy in the second regime would lead to indeterminacy if the regime were treated in isolation. With $\phi_{2}=0.9951$, however, the generalized Taylor principle of Davig and Leeper (2006) implies that the MSV solution would be the only stationary solution. With

\footnotetext{
${ }^{7}$ The assumption that a process is stationary in each regime is not enough to ensure overall stationarity. One can easily modify our example to produce a solution that is stationary for each regime in isolation, but non-stationary for the system that allows regimes to switch.
} 

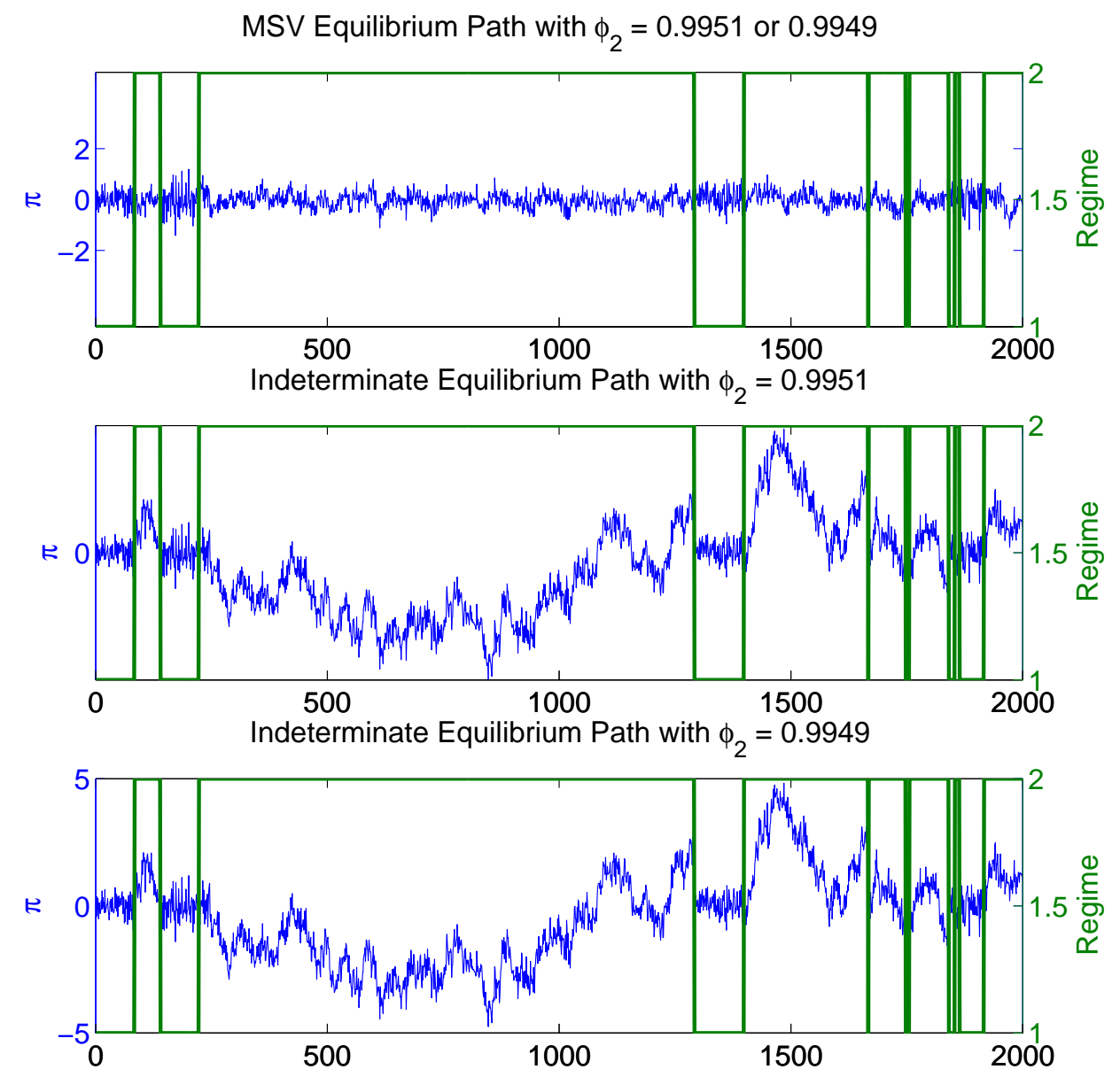

FiguRE 1. Simulated paths of inflation (deviations from the target) with $\phi_{1}=2.2, p_{1,1}=0.98, p_{2,2}=0.995$.

$\phi_{2}=0.9949$, their uniqueness condition is violated. However, using the mean-squarestability criterion, the indeterminate solutions are stationary in both cases. ${ }^{8}$

It is worth noting that the paths associated with the policies $\phi_{2}=0.9951$ and $\phi_{2}=0.9949$ are hard to tell apart even though one violates the generalized Taylor principle and the other does not. In both cases, inflation in the indeterminate

\footnotetext{
${ }^{8}$ We calibrated this example by setting $\varepsilon_{t}$ to be $N(0,1)$ and $\nu_{t}$ to be $N(0,0.01)$. The values of $\frac{\kappa_{1}}{\phi_{1}}$ and $\frac{\kappa_{2}}{\phi_{2}}$ are 0.4 and 0.2 so that the size of the fundamental shock in the first regime is twice the size in the second regime. The initial condition is $\pi_{1}=g_{1} r_{1}+\frac{\kappa_{1}}{\phi_{1}} \varepsilon_{1}$. All the paths are based on the same sequence of the real interest rate $r_{t}$ and the same sequence of fundamental shocks $\varepsilon_{t}$. The non-fundamental shock $\gamma_{t}$ is set to $\frac{\kappa_{2}}{\phi_{2}} \varepsilon_{t}$ when the second regime occurs and set to zero when the first regime occurs. Therefore, in the indeterminate equilibrium the size of the random shock is the same for both regimes. These assumptions allow us to isolate the effects of indeterminacy from the the effects of different shock variances across regimes.
} 
equilibrium is much more persistent than that in the MSV equilibrium. This is consistent with Clarida, Galí, and Gertler's (2000) interpretation of the U.S. data. They attribute the serially-dependent and volatile behavior of inflation in the 1970s to indeterminate monetary policy and the remarkable reduction in volatility and serial dependence of the Volcker-Greenspan years to the successful application of a Taylor rule that implements an MSV equilibrium. In both examples presented in Figure 1, the stochastic process that describes equilibrium is stable around the steady state. This is precisely our definition of stationarity for MSRE models.

Now we turn to our second example which satisfies the more restricted definition of boundedness favored by Davig and Leeper. Here we set $\phi_{1}=24 / 9$ and $\phi_{2}=-1 / 3$ with $p_{11}=0.01$ and $p_{22}=0.5$. The policy maker in the first regime is an inflation hawk, while monetary policy in the second regime would lead to indeterminacy if this regime were absorbing. If the structural shocks $\left\{\varepsilon_{t}\right\}$ are bounded, the indeterminate solution represented by Eq. (18) will be bounded as well. Hence, this example satisfies the uniqueness criterion of Davig and Leeper (2006) which claims to establish an equivalence between the existence of a unique bounded solution to an MSRE model and the generalized Taylor principle derived from the linear RE counterpart. Our example demonstrates that a positive value of $\phi_{i}$ in both regimes is essential for this result to hold. If this condition breaks down, as may happen in the planning solution discussed by Rotemberg and Woodford (1999a, Page 83), there will exist sunspot equilibria in general whenever there is a sunspot equilibrium in at least one regime considered in isolation. This example raises serious questions about the validity of the generalized Taylor principle for MSRE models.

\section{CONCLUSION}

We have shown in this paper that the distinction between linear and MSRE models is important but its consequences for equilibrium are not well understood. We have demonstrated that the properties of uniqueness, stationarity and boundedness, even for the simple MSRE model of monetary policy studied in this paper, are fundamentally different from those of linear models. Characterizing the full class of equilibria remains a challenging task. Contrary to the existing literature, we have shown that multiple equilibria are more prevalent than commonly thought and that the dynamic behavior of equilibrium sample paths depends in subtle ways on both the current and the past realized regimes. Regrettably, the generalized Taylor principle does not hold universally, even for a simple MSRE model. 


\section{Appendix A. Proof of Proposition 3}

Substituting Eq. (7) into Eq. (8) we have,

$$
\pi_{t}=g_{\xi_{t}} r_{t}+\frac{1}{\phi_{\xi_{t}}} E_{t}\left[\pi_{t+1}-g_{\xi_{t+1}} r_{t+1}\right]+\frac{\kappa_{\xi_{t}}}{\phi_{\varepsilon_{t}}} \varepsilon_{t}
$$

Substituting for $r_{t+1}$ from (4) and collecting terms leads to

$$
\pi_{t}=\frac{1}{\phi_{\xi_{t}}} E_{t}\left[\pi_{t+1}\right]+\frac{1}{\phi_{\xi_{t}}} r_{t}\left[\phi_{\xi_{t}} g_{\xi_{t}}-\rho\left(p_{1, \xi_{t}} g_{1}+p_{2, \xi_{t}} g_{2}\right)\right]+\frac{\kappa_{\xi_{t}}}{\phi_{\xi_{t}}} \varepsilon_{t} .
$$

or since

$$
\begin{gathered}
p_{1, \xi_{t}} g_{1}+p_{2, \xi_{t}} g_{2} \equiv E_{t} g_{\xi_{t+1}} \\
\pi_{t}=\frac{1}{\phi_{\xi_{t}}} E_{t}\left[\pi_{t+1}\right]+\frac{1}{\phi_{\xi_{t}}} r_{t}\left[\phi_{\xi_{t}} g_{\xi_{t}}-\rho E_{t} g_{\xi_{t+1}}\right]+\frac{\kappa_{\xi_{t}}}{\phi_{\xi_{t}}} \varepsilon_{t}
\end{gathered}
$$

Note that if $\xi_{t}=1$, it follows from (5) that

$$
\left(\phi_{1}-\rho p_{1,1}\right) g_{1}-\rho p_{2,1} g_{2}=1 \text {. }
$$

Similarly, if $\xi_{t}=2$, it follows from (5) that

$$
-\rho p_{1,2} g_{1}+\left(\phi_{2}-\rho p_{2,2}\right) g_{2}=1 \text {. }
$$

Hence we have

$$
\pi_{t}=\frac{1}{\phi_{\xi_{t}}} E_{t}\left[\pi_{t+1}\right]+\frac{1}{\phi_{\xi_{t}}} r_{t}+\frac{\kappa_{\xi_{t}}}{\phi_{\xi_{t}}} \kappa_{\xi_{t}} \varepsilon_{t}
$$

which is equivalent to $(3)$.

\section{Appendix B. Proof of Proposition 4}

We first show that $\eta_{t+1}$ has zero conditional mean. If $\xi_{t}=1$,

$$
E_{t}\left[\eta_{t+1}\right]=p_{1,1} \frac{\kappa_{1}}{\phi_{1}} E_{t}\left[\varepsilon_{t+1}\right]+p_{2,1}\left(E_{t}\left[\gamma_{t+1}\right]+\frac{\kappa_{2}}{\phi_{2}} E_{t}\left[\varepsilon_{t+1}\right]\right)=0
$$

and if $\xi_{t}=2$,

$$
\begin{aligned}
E_{t}\left[\eta_{t+1}\right]=p_{1,2}\left(\frac{\kappa_{1}}{\phi_{1}} E_{t}\left[\varepsilon_{t+1}\right]\right. & \left.-\frac{1}{a_{2}}\left(x_{t}-\frac{\kappa_{2}}{\phi_{2}} \varepsilon_{t}\right)\right) \\
+ & p_{2,2}\left(E_{t}\left[\gamma_{t+1}\right]+\frac{\kappa_{2}}{\phi_{2}} E_{t}\left[\varepsilon_{t+1}\right]+\phi_{2} \frac{p_{1,2}}{p_{2,2}}\left(x_{t}-\frac{\kappa_{2}}{\phi_{2}} \varepsilon_{t}\right)\right)=0 .
\end{aligned}
$$

Next, we derive the solution to Eq. (8). Given $x_{1}$, the sequence $\left\{x_{t+1}\right\}_{t=1}^{\infty}$ can be constructed recursively from the following transition equations, which are derived by using Eq. (9) and Definition (15). When $\xi_{t}=1$,

$$
x_{t+1}= \begin{cases}\frac{1}{a_{1}}\left(x_{t}-\frac{\kappa_{1}}{\phi_{1}} \varepsilon_{t}\right)+\frac{\kappa_{1}}{\phi_{\kappa} \xi_{t}} \varepsilon_{t+1}, & \text { if } \xi_{t+1}=1, \\ \frac{1}{\phi_{\varepsilon_{t}}}\left(x_{t}-\frac{\kappa_{1}}{\phi_{1}} \varepsilon_{t}\right)+\gamma_{t+1}+\frac{\kappa_{2}}{\phi_{2}} \varepsilon_{t+1}, & \text { if } \xi_{t+1}=2\end{cases}
$$


and when $\xi_{t}=2$,

$$
x_{t+1}= \begin{cases}\frac{\kappa_{1}}{\phi_{1}} \varepsilon_{t+1}, & \text { if } \xi_{t+1}=1, \\ \phi_{2}\left(x_{t}-\frac{\kappa_{2}}{\phi_{2}} \varepsilon_{t}\right)+\gamma_{t+1}+\frac{\kappa_{2}}{\phi_{2}} \varepsilon_{t+1}+\phi_{2} \frac{p_{1,2}}{p_{2,2}}\left(x_{t}-\sigma_{2} \varepsilon_{t}\right), & \text { if } \xi_{t+1}=2 .\end{cases}
$$

Since we have imposed the initial condition $x_{1}=\frac{\kappa_{1}}{\phi_{1}} \varepsilon_{1}$, it follows by induction from Eqs. (A1) and (A2) that when $\xi_{t+1}=1, x_{t+1}=\frac{\kappa_{1}}{\phi_{1}} \varepsilon_{t+1}$ for all $t \geq 1$. The transition from state 1 to state 2 can be simplified using this initial condition, and Eq. (A1) can be written as the expression (16). Eq. (A2) can be also simplified, using the fact that $p_{1,2}+p_{2,2}=1$, to yield the expression (17). The initial condition (14) and Eqs. (16) and (17) completely characterize the evolution of $x_{t}$ for $t=1, \ldots \infty$.

Finally, we show that $\lim _{s \rightarrow \infty} E_{t}\left[x_{t+s}\right]=0$. Using Eqs. (16) and (17), we obtain the following expression:

$$
E_{t}\left[x_{t+1}\right]= \begin{cases}0, & \text { if } \xi_{t}=1 \\ \phi_{2}\left(x_{t}-\frac{\kappa_{2}}{\phi_{2}} \varepsilon_{t}\right), & \text { if } \xi_{t}=2\end{cases}
$$

A simple induction argument, again using Eqs. (16) and (17) shows that,

$$
E_{t}\left[x_{t+s}\right]=E_{t}\left[E_{t+1}\left[x_{t+k}\right]\right]= \begin{cases}0, & \text { if } \xi_{t}=2 \\ \left(\phi_{2}\right)^{s}\left(x_{t}-\frac{\kappa_{2}}{\phi_{2}} \varepsilon_{t}\right), & \text { if } \xi_{t}=2\end{cases}
$$

Because $\left|\phi_{2}\right|<1, \lim _{s \rightarrow \infty} E_{t}\left[x_{t+s}\right]=0$. Since $\left\{\gamma_{t+1}\right\}$ is arbitrary, we have completed the proof that $\left\{\left\{\eta_{t+1}\right\}_{t=1}^{\infty}, x_{1}\right\}$ generates multiple solutions.

\section{Appendix C. Proof of Proposition 5}

Theorem 3.33 of Costa, Fragoso, and Marques (2004) implies that, if the Markov chain is ergodic, we need only show that solutions given by (14), (16), and (17) are mean-square stable when $\varepsilon_{t}$ and $\gamma_{t}$ are zero. By Theorem 3.9 of CFM, the stability condition is equivalent to showing that there exist constants $1 \leq \beta<\infty$ and $0<\zeta<1$ such that

$$
E_{1}\left[x_{t}^{2}\right] \leq \beta \zeta^{t-1} x_{1}^{2}
$$

for $t \geq 1$. When $\varepsilon_{t}$ and $\gamma_{t}$ are zero, $x_{t}$ will be non-zero only if $\xi_{1}=\cdots=\xi_{t}=2$. In this case, $x_{t}=\left(\frac{1}{a_{2} p_{2,2}}\right)^{t-1} x_{1}$ and this event occurs with probability $p_{2,2}^{t-1}$. Thus

$$
\begin{aligned}
E_{1}\left[x_{t}^{2}\right] & =p_{2,2}^{t-1}\left(\frac{\phi_{2}}{p_{2,2}}\right)^{2(t-1)} x_{1}^{2}, \\
& =\left(\frac{\phi_{2}}{\sqrt{p_{2,2}}}\right)^{2 t-2} x_{1}^{2} .
\end{aligned}
$$

The results now follow from the assumption that $\left|\frac{\phi_{2}}{\sqrt{p_{2,2}}}\right|<1$. 


\section{REFERENCES}

Clarida, R., J. Galí, And M. Gertler (2000): "Monetary Policy Rules and Macroeconomic Stability: Evidence and Some Theory," Quarterly Journal of Economics, 115(1), 147-180.

Costa, O., M. Fragoso, And R. Marques (2004): Discrete-Time Markov Jump Linear Systems. Springer, New York.

Davig, T., And E. Leeper (2005): "Generalizing the Taylor Principle," NBER Working Paper 11874 .

(2006): "Generalizing the Taylor Principle," Mimeo: University of Indiana.

Farmer, R. E. A., D. F. Waggoner, And T. Zha (2006): "Minimal State Variable Solutions to Markov-Switching Rational Expectations Models," UCLA mimeo.

Hamilton, J. D. (1994): Times series analsis. Princeton University Press, Princeton, NJ.

KInG, R. G. (2000): "The New IS-LM Model: Language, Logic, and Limits," Federal Reserve Bank of Richmond Economic Quarterly, 86/3, 45-103.

Rotemberg, J., And M. Woodford (1999a): Monetary Policy Ruleschap. 2, pp. 57-126. NBER.

RotemberG, J. J., AND M. Woodford (1999b): "Interest Rate Rules in an Estimated Sticky Price Model," in Monetary Policy Rules, ed. by J. B. Taylor, pp. 57-119. University of Chicago Press, Chicago.

Sims, C. A. (2001): "Solving Linear Rational Expectations Models," Journal of Computational Economics, 20(1-2), 1-20.

Svensson, L., And N. Williams (2005): "Monetary Policy with Model Uncertainty: Distribution Forecast Targeting," Princeton University Mimeo.

Woodford, M. (2003): Interest and Prices: Foundations of a Theory of Monetary Policy. Princeton University Press, Princeton, N.J.

UCla, Federal Reserve Bank of Atlanta, Federal Reserve Bank of Atlanta 